\title{
Osteopontin promotes host defense during Klebsiella pneumoniae-induced pneumonia
}

\author{
G.J.W. van der Windt*\#, J.J. Hoogerwerf*,\#, A.F. de Vos*,\#, S. Florquin and \\ T. van der Poll*,\#
}

ABSTRACT: Klebsiella pneumoniae is a common cause of nosocomial pneumonia. Osteopontin (OPN) is a phosphorylated glycoprotein involved in inflammatory processes, some of which is mediated by CD44. The aim of this study was to determine the role of OPN during $K$. pneumoniaeinduced pneumonia.

Wild-type (WT) and OPN knockout (KO) mice were intranasally infected with $10^{4}$ colony forming units of $K$. pneumoniae, or administered Klebsiella lipopolysaccharides (LPS). In addition, recombinant OPN (rOPN) was intranasally administered to WT and CD44 KO mice.

During Klebsiella pneumonia, WT mice displayed elevated pulmonary and plasma OPN levels. OPN KO and WT mice showed similar pulmonary bacterial loads $6 \mathrm{~h}$ after infection; thereafter, Klebsiella loads were higher in lungs of OPN KO mice and the mortality rate in this group was higher than in WT mice. Early neutrophil recruitment into the bronchoalveolar space was impaired in the absence of OPN after intrapulmonary delivery of either Klebsiella bacteria or Klebsiella LPS. Moreover, rOPN induced neutrophil migration into the bronchoalveolar space, independent from CD44. In vitro, OPN did not affect $K$. pneumoniae growth or neutrophil function.

In conclusion, OPN levels were rapidly increased in the bronchoalveolar space during $K$. pneumoniae pneumonia, where OPN serves a chemotactic function towards neutrophils, thereby facilitating an effective innate immune response.

KEYWORDS: Infection, Klebsiella pneumoniae, lung, mice, osteopontin

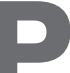

neumonia is a common and serious illness that is a major cause of morbidity and mortality in humans and Klebsiella pneumoniae is a frequently isolated causative pathogen in nosocomial pneumonia [1].

Osteopontin (OPN) is a phosphorylated glycoprotein, expressed by a broad range of tissues and cells, which is involved in a number of physiological and pathological processes. OPN has been implicated as an important regulator of inflammation, occupying a central role in both innate and adaptive immunity by mediating inflammatory cell differentiation, maturation and migration, and cytokine production. Some of these processes are mediated through one of its receptors, the transmembrane molecule, CD44, which is known to affect cellular migration and chemokine responses [2]. Animal studies have especially pointed to a role for OPN in lung inflammation, such as seen in allergy and asthma [3, 4], acute respiratory distress syndrome [5], fibrosis [6] and parasitic infection [7]. Furthermore, patients suffering from diverse pulmonary diseases, including tuberculosis, silicosis and sarcoidosis, displayed enhanced OPN expression in their lungs [5, 7], and patients with tuberculosis or interstitial pneumonia had dramatically elevated plasma OPN levels compared with healthy controls [8,9]. Recently, plasma OPN concentrations were found to be increased in patients with sepsis, the majority of whom suffered from pneumonia as the primary source of infection [10].

Considering the association between OPN expression and pulmonary disease and sepsis, we sought to determine the potential role of OPN in the host response to lower respiratory tract infection caused by K. pneumoniae. We show for the first time that OPN is important for early neutrophil recruitment to the bronchoalveolar space and thereby for an effective immune response during K. pneumoniae-induced pneumonia.

\section{MATERIALS AND METHODS}

Mice

C57BL/6 wild-type (WT) mice were purchased from Harlan Sprague Dawley Inc. (Horst, The Netherlands). OPN knockout (KO) mice (Jackson Laboratories, Bar Harbor, ME) and CD44 KO mice (kindly provided by A. Berns, Netherlands Cancer Institute, Amsterdam, The Netherlands) [11], both

\section{AFFILIATIONS}

${ }^{*}$ Center of Infection and Immunity Amsterdam (CINIMA), University of Amsterdam,

${ }^{*}$ Center for Experimental and Molecular Medicine, University of Amsterdam, and

'Dept of Pathology, Academic Medical Center, University of Amsterdam, Amsterdam, The Netherlands.

CORRESPONDENCE

T. van der Poll

Academic Medical Center Meibergdreef 9 Room G2-130 1105 AZ Amsterdam The Netherlands E-mail: t.vanderpoll@amc.uva.nl

Received: Jan 062010 Accepted after revision: March 272010 First published online: April 082010 
with a C57BL/ 6 genetic background, were bred in the animal facility of the Academic Medical Center (Amsterdam).

\section{Study design}

The Animal Care and Use Committee of the University of Amsterdam (Amsterdam) approved all experiments. Pneumonia was induced by intranasal inoculation of $10^{4}$ colony-forming units (CFU) of K. pneumoniae serotype 2 (ATCC 43816; American Type Culture Collection, Rockville, MD, USA) as described [12]. Sample harvesting and processing, and determinations of bacterial loads and cell counts were performed as described [12]. In some experiments, K. pneumoniae lipopolysaccharides (LPS) (100 $\mu$; L1519, Sigma, St. Louis, MO, USA) or recombinant mouse OPN (rOPN; 1 or $10 \mu \mathrm{g}$; $<1.0 \mathrm{EU}$ endotoxin per $1 \mu \mathrm{g}$ as determined by the Limulus amoebocyte lysate assay; R\&D Systems, Minneapolis, MN, USA) was administered intranasally. For neutralisation of keratinocyte-derived cytokine (KC), $50 \mu \mathrm{g}$ of monoclonal anti-murine KC antibody (R\&D Systems) was injected intraperitoneally $2 \mathrm{~h}$ before rOPN, as described [13]; and control animals received isotype control antibody (R\&D Systems).

\section{Assays}

Myeloperoxidase (MPO), OPN, KC, lipopolysaccharide (LPS)induced CXC chemokine (LIX) and macrophage inflammatory protein (MIP)-2 levels were determined by ELISA (MPO: Hycult, Uden, The Netherlands; others: R\&D Systems, Abingdon, UK). Tumour necrosis factor (TNF)- $\alpha$ and interleukin (IL)- 6 were measured by cytometric bead array multiplex assay (BD Biosciences, San Jose, CA, USA). Aspartate aminotransferase (ASAT), alanine aminotransferase (ALAT), urea and creatinin were determined with commercially available kits (Sigma), using a Hitachi analyser (Roche) according to the manufacturers' instructions.

\section{Immunohistochemistry}

Paraffin lung sections were stained with haematoxylin and eosin or Ly-6G monoclonal antibody (BD Pharmingen, San Diego, CA, USA) and semi-quantitatively scored as described [14].

\section{Bactericidal assay, phagocytosis and phago-lysosomal fusion}

K. pneumoniae was cultured in the presence of $800-0.8 \mathrm{ng} \cdot \mathrm{mL}^{-1}$ rOPN or in tryptic soy broth only. At indicated time points, the number of bacteria was determined by plating on blood-agar plates and counting colonies after $16 \mathrm{~h}$ of incubation at $37^{\circ} \mathrm{C}$. Neutrophil phagocytosis of K. pneumoniae was determined as described [15]. In order to determine phago-lysosomal fusion in neutrophils after phagocytosis of $K$. pneumoniae, the procedure was similar except that K. pneumoniae was labelled with pHrodo (Invitrogen, Breda, The Netherlands) and neutrophils being labelled using anti-Gr-1-FITC (BD Pharmingen) [16]. Phagocytosis and phago-lysosomal fusion index of each sample was calculated: mean fluorescence of positive cells $\times \%$ positive cells.

\section{Statistical analysis}

All data are expressed as mean \pm SEM. Comparisons for two groups were done with Mann-Whitney U-tests, comparisons for more than two groups were done with Kruskall-Wallis followed by Dunn's Multiple Comparison tests. Numbers of surviving animals at the end of observation period were compared by the Chi-squared test. These analyses were performed using GraphPad Prism version 4.0, GraphPad Software (San Diego, CA). A p-value $<0.05$ was considered statistically significant.

\section{RESULTS \\ Osteopontin levels are increased during K. pneumoniae infection}

To obtain a first insight into a potential role for OPN during gram-negative pneumonia, we measured OPN concentrations in bronchoalveolar lavage fluid (BALF), lung and plasma from WT mice before infection and 6, 24 and $48 \mathrm{~h}$ after infection with $10^{4}$ CFU K. pneumoniae. OPN concentrations in BALF increased rapidly and peaked at $6 \mathrm{~h}$ after infection (fig. 1a). Lung OPN levels increased more slowly and were significantly elevated compared with baseline from $24 \mathrm{~h}$ onward (fig. 1b). Plasma levels were increased already at $6 \mathrm{~h}$ after K. pneumoniae infection compared with baseline and continued to rise up to $48 \mathrm{~h}$ (fig. 1c).

\section{Osteopontin limits pulmonary bacterial growth}

To determine whether OPN affected mortality due to Klebsiella pneumonia, we followed WT and OPN KO mice for 14 days after K. pneumoniae infection. Although median survival times did not differ, a significant proportion of WT mice $(27 \%)$ survived the observation period whereas none of the WT mice did ( $p=0.03$; fig. 2). Next, we questioned whether OPN affects the antibacterial response against K. pneumoniae. Therefore, we determined bacterial outgrowth in BALF and lung homogenates of WT and OPN KO mice 6, 24 and $48 \mathrm{~h}$ after infection. At $6 \mathrm{~h}$ after infection, bacterial loads were similar in BALF and lungs from both mouse strains (fig. $3 \mathrm{a}$ and b). At 24 and $48 \mathrm{~h}$ after infection, however, bacterial loads were 10-100-fold higher in BALF and lungs of OPN KO compared with WT mice (BALF: $\mathrm{p}<0.01$; lung: $\mathrm{p}<0.05$ for both time points; fig. $3 \mathrm{a}$ and $b)$. To obtain insight into the dissemination of the infection, we determined bacterial loads in blood and spleen. At $6 \mathrm{~h}$, bacteria were not detectable yet in these organs, whereas after 24 and $48 \mathrm{~h}$, bacterial loads were similar in blood and spleen from WT and OPN KO mice (fig. $3 \mathrm{c}$ and d). In line, neither plasma cytokine levels (TNF- $\alpha$ and IL-6) nor liver injury (as indicated by ASAT and ALAT plasma levels) and kidney injury (as indicated by urea and creatinin plasma levels) were different in OPN KO mice compared with WT mice (table 1). Together, these data suggest that OPN contributes to local host defence, within the pulmonary compartment, during Klebsiella pneumonia.

\section{Osteopontin deficiency does not influence pulmonary pathology}

To obtain insight into the role of OPN in pulmonary inflammation in response to K. pneumoniae, we analysed lung histology slides obtained 6, 24 or $48 \mathrm{~h}$ after infection using a semi-quantitative scoring system. Already at $6 \mathrm{~h}$ after infection, mild interstitial inflammation and pleuritis were found in all mice. These parameters were dramatically increased at 24 and $48 \mathrm{~h}$, and bronchitis, endothelialitis and oedema were also present from $24 \mathrm{~h}$ onward. Importantly, the extent of lung inflammation was similar in both groups at all time points (fig. 4). In addition, lung weights did not differ at $24 \mathrm{~h}$; 

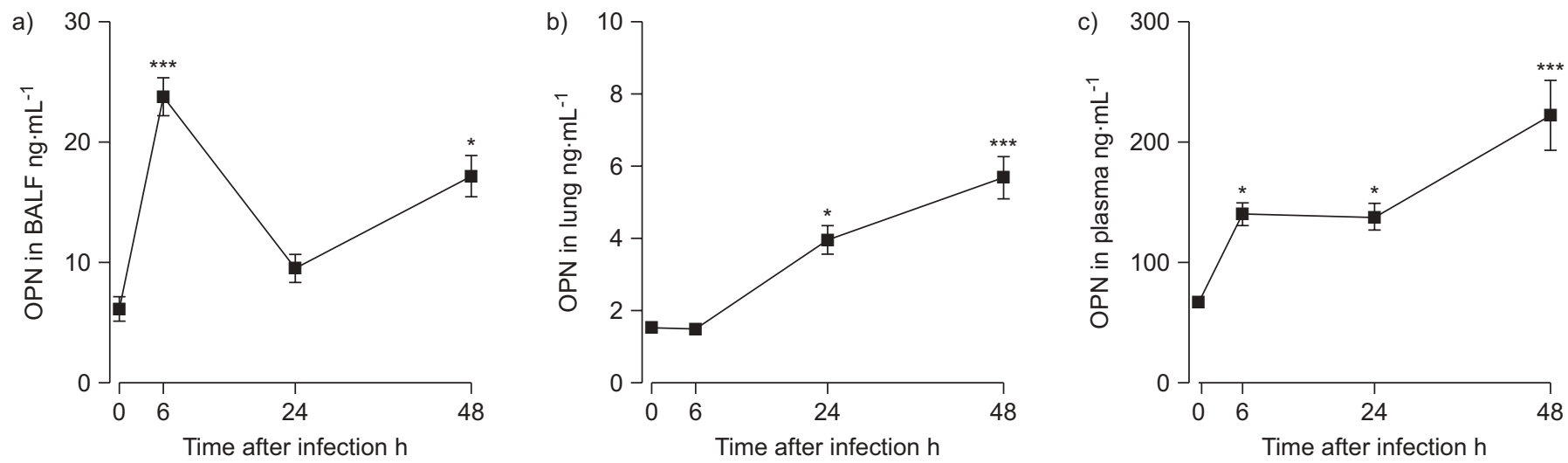

FIGURE 1. Pulmonary and plasma osteopontin (OPN) concentrations are elevated during Klebsiella pneumoniae infection. OPN concentrations in a) bronchoalveolar lavage fluid (BALF), b) lung and c) plasma before (0), 6, 24 and $48 \mathrm{~h}$ after infection with $10^{4}$ colony-forming units of $K$. pneumoniae. Data are presented as mean \pm sEM; $\mathrm{n}=8$ mice per group. *: $p<0.05 ; * \star *: p<0.001$ compared with $0 \mathrm{~h}$.

however, lungs from OPN KO mice were significantly heavier compared with lungs from WT mice at $48 \mathrm{~h}$ after induction of pneumonia (24 h: $115 \pm 2$ versus $109 \pm 3 ; 48$ h: $109 \pm 5$ versus $134 \pm 9$, for WT versus OPN KO mice, respectively; $\mathrm{p}=0.13$ and $\mathrm{p}<0.01$, respectively).

\section{Osteopontin deficiency results in impaired early neutrophil recruitment into the bronchoalveolar space upon $K$. pneumoniae infection}

As pulmonary bacterial loads were similar at $6 \mathrm{~h}$ after infection but enhanced in the absence of OPN from $24 \mathrm{~h}$ onward, we next questioned whether OPN affects the early neutrophil recruitment to the lungs upon $K$. pneumoniae infection. At $6 \mathrm{~h}$ neutrophil numbers in BALF were significantly reduced in OPN KO mice as compared to WT mice (fig. $5 a, p<0.05$ ). In lung tissue, however, neutrophil infiltration was similar in WT and OPN KO mice, as determined by MPO measurements in lung homogenates and quantification of Ly6+ cells in lung tissue slides (fig. $5 b$ and c). Of interest, at this early time point, OPN levels were strongly increased in BALF but not in lung homogenates compared with uninfected mice (fig. 1).

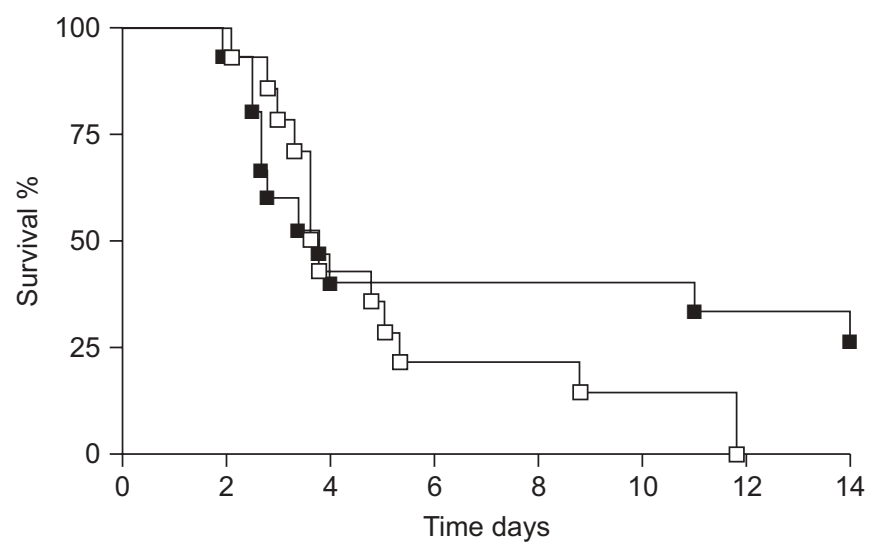

FIGURE 2. Osteopontin (OPN) knockout (KO) mice show enhanced mortality. Percentage survival of wild-type ( $\mathbf{\square})$ and OPN KO $(\square)$ mice until 14 days after intranasal infection with $10^{4}$ colony-forming units of Klebsiella pneumoniae. $n=14-15$ per group. $p=0.03$ between groups.
As chemokines play an important role in the recruitment of inflammatory cells, we measured CXC chemokine levels in BALF at $6 \mathrm{~h}$ after infection. Whereas KC levels were significantly decreased in OPN KO mice compared with WT mice, MIP-2 and LIX concentrations were similar (fig. 5d-f). Of note, at 24 and $48 \mathrm{~h}$ after infection, neutrophil numbers in BALF were significantly enhanced in OPN KO mice compared with WT mice $\left(24 \mathrm{~h}: 6.39 \pm 0.7\right.$ versus $15.3 \pm 1.7 \times 10^{5}$ cells $\cdot \mathrm{mL}^{-1}$ BALF; and $48 \mathrm{~h}: 5.1 \pm 0.6$ versus $13.2 \pm 3.3 \times 10^{5}$ cells $\cdot \mathrm{mL}^{-1}$ BALF; $\mathrm{p}<0.01$ and $\mathrm{p}<0.05$, respectively); similarly, chemokine levels were higher in BALF from OPN KO mice than in BALF from WT mice at these later time points (data not shown). Importantly, pulmonary bacterial loads were 10-100-times higher in OPN KO mice from $24 \mathrm{~h}$ on (fig. 3), probably causing the enhanced proinflammatory response in these mice at these later time points. Together, these data suggest that OPN present in BALF contributes to the influx of neutrophils into the bronchoalveolar space early after pulmonary infection by K. pneumoniae.

\section{Osteopontin deficiency results in reduced early neutrophil influx into the bronchoalveolar space after intrapulmonary delivery of Klebsiella LPS}

To obtain further proof for a role for OPN in early neutrophil recruitment during gram-negative lung inflammation without the possible influence of different bacterial loads, we instilled Klebsiella LPS via the airways of OPN KO and WT mice and determined the numbers of neutrophils in BALF harvested 6 and $24 \mathrm{~h}$ later. In WT mice, LPS administration caused a significant rise in the BALF levels of OPN at $6 \mathrm{~h}$ after LPS $(\mathrm{p}<0.05)$; whereas, at $24 \mathrm{~h}$, OPN levels returned to baseline (fig. 6a). Like in mice infected with viable Klebsiella, OPN KO mice administered with Klebsiella LPS displayed fewer neutrophils in their BALF compared with WT mice at $6 \mathrm{~h}$ but not at $24 \mathrm{~h}$ after LPS (fig. 6b). Importantly, OPN concentrations were elevated in WT mice at $6 \mathrm{~h}$ but not at $24 \mathrm{~h}$ after LPS; thus these data further indicate that OPN present in BALF contributes to the influx of neutrophils into the bronchoalveolar space.

Osteopontin is chemotactic in the bronchoalveolar space To determine whether OPN is indeed chemotactic in the bronchoalveolar space we intranasally instilled PBS or 1 or 

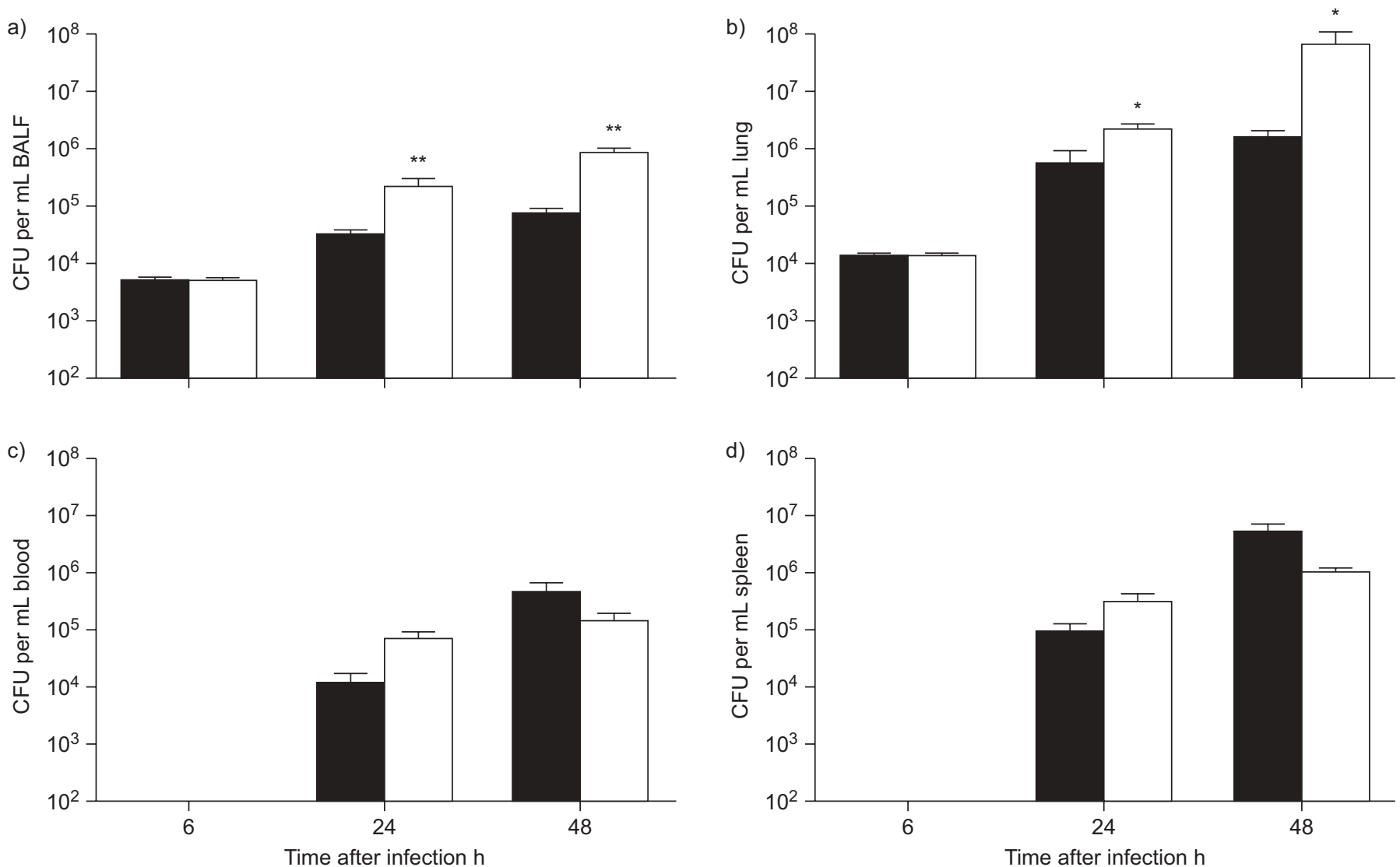

FIGURE 3. Enhanced bacterial outgrowth in osteopontin (OPN) knockout (KO) mice. Wild-type (WT) ( $\mathbf{\square})$ and OPN KO ( $\square$ ) mice were infected with $10^{4}$ colony-forming units (CFU) of Klebsiella pneumoniae and bacterial loads were determined 6, 24 and $48 \mathrm{~h}$ after infection in a) bronchoalveolar lavage fluid (BALF), b) lung, c) blood and d) spleen. CFU in blood and spleen was not detectable at $6 \mathrm{~h}$. Data are presented as mean \pm SEM; $n=8$ mice per group. *: $p<0.05 ;{ }^{* *}$ : $p<0.01$ compared with WT mice.

$10 \mu \mathrm{g}$ of rOPN in WT mice. At $6 \mathrm{~h}$ after instillation we determined the numbers of infiltrated cells in BALF. Although $1 \mu \mathrm{g}$ of rOPN was not associated with influx of cells, instillation

\begin{tabular}{|c|c|c|c|c|}
\hline TABLE 1 & $\begin{array}{l}\text { o differenc } \\
\text { oncentratic } \\
\text { id osteop }\end{array}$ & $\begin{array}{l}\text { in plasma } \\
\text { and orgar } \\
n(\mathrm{OPN})\end{array}$ & $\begin{array}{l}\text { ytokine } \\
\text { ailure in w } \\
\text { ckout ( } \mathrm{K}\end{array}$ & $\begin{array}{l}\text { type (WT) } \\
\text { mice }\end{array}$ \\
\hline \multirow{2}{*}{$\begin{array}{l}\text { Plasma } \\
\text { mediators }\end{array}$} & \multicolumn{2}{|c|}{$24 \mathrm{~h}$} & \multicolumn{2}{|c|}{$48 \mathrm{~h}$} \\
\hline & WT & OPN KO & WT & OPN KO \\
\hline TNF- $\alpha \mathrm{pg} \cdot \mathrm{mL}^{-1}$ & $66.6 \pm 18.4$ & $93.3 \pm 22.0$ & $44.2 \pm 8.0$ & $35.2 \pm 7.7$ \\
\hline $\mathrm{IL}-6 \mathrm{pg} \cdot \mathrm{mL}^{-1}$ & $264 \pm 72$ & $403 \pm 112$ & $353 \pm 120$ & $193 \pm 34$ \\
\hline ALAT U. $L^{-1}$ & $17.6 \pm 2.2$ & $17.2 \pm 3.3$ & $666 \pm 212$ & $325 \pm 143$ \\
\hline ASAT U.L-1 & $60.0 \pm 7.4$ & $50.6 \pm 1.7$ & $1125 \pm 422$ & $659 \pm 332$ \\
\hline Urea $\mathrm{mmol} \cdot \mathrm{L}^{-1}$ & $4.6 \pm 0.4$ & $5.7 \pm 0.3$ & $6.3 \pm 0.2$ & $6.6 \pm 0.4$ \\
\hline $\begin{array}{l}\text { Creatinin } \\
\mu \mathrm{mol} \cdot \mathrm{L}^{-1}\end{array}$ & $7.6 \pm 0.5$ & $6.7 \pm 0.2$ & $7.8 \pm 0.6$ & $8.0 \pm 0.7$ \\
\hline
\end{tabular}

Data are presented as mean \pm SEM. Plasma concentrations of tumour necrosis factor (TNF)- $\alpha$, interleukin (IL)- 6 , alanine aminotransferase (ALAT), aspartate aminotransferase (ASAT), urea and creatinin in WT and OPN KO mice measured at 24 and $48 \mathrm{~h}$ after intranasal infection with $10^{4}$ CFU Klebsiella pneumoniae. Data are expressed as mean \pm SEM. $n=6-8$ mice per group. of $10 \mu \mathrm{g}$ rOPN resulted in the recruitment of neutrophils to the bronchoalveolar space (fig. 6c, $p<0.001$ compared with PBS). Since the reduced neutrophil numbers in OPN KO mice early after K. pneumoniae infection were accompanied by reduced KC concentrations, we determined whether the chemotactic effect of rOPN was $\mathrm{KC}$ dependent. Therefore, we pre-treated mice with an a neutralising anti-KC or control antibody (as previously described $[13,17]) 2 \mathrm{~h}$ prior to intrapulmonary delivery of rOPN $(10 \mu \mathrm{g})$. Anti-KC treatment did not affect neutrophil recruitment into BALF induced by $\mathrm{rOPN}$ (fig. 6d). CD44 is a transmembrane molecule mediating cellular migration and chemokine responses [18], and an important receptor for OPN [19]. Therefore, we determined whether neutrophil recruitment induced by OPN was CD44 dependent. Upon $10 \mu \mathrm{g}$ of rOPN administration WT and CD44 KO mice demonstrated similar neutrophil recruitment after $6 \mathrm{~h}$ (fig. 6e). Together, these data suggest that OPN is chemotactic in the lung by a mechanism that is independent from KC and CD44.

\section{Osteopontin does not affect K. pneumoniae growth and neutrophil phagocytosis or phago-lysosomal fusion}

The aforementioned experiments established that in the absence of OPN, mice display diminished early recruitment of neutrophils to the bronchoalveolar space, accompanied by increased bacterial loads. Apart from impaired early neutrophil influx, the enhanced bacterial outgrowth in the absence of 

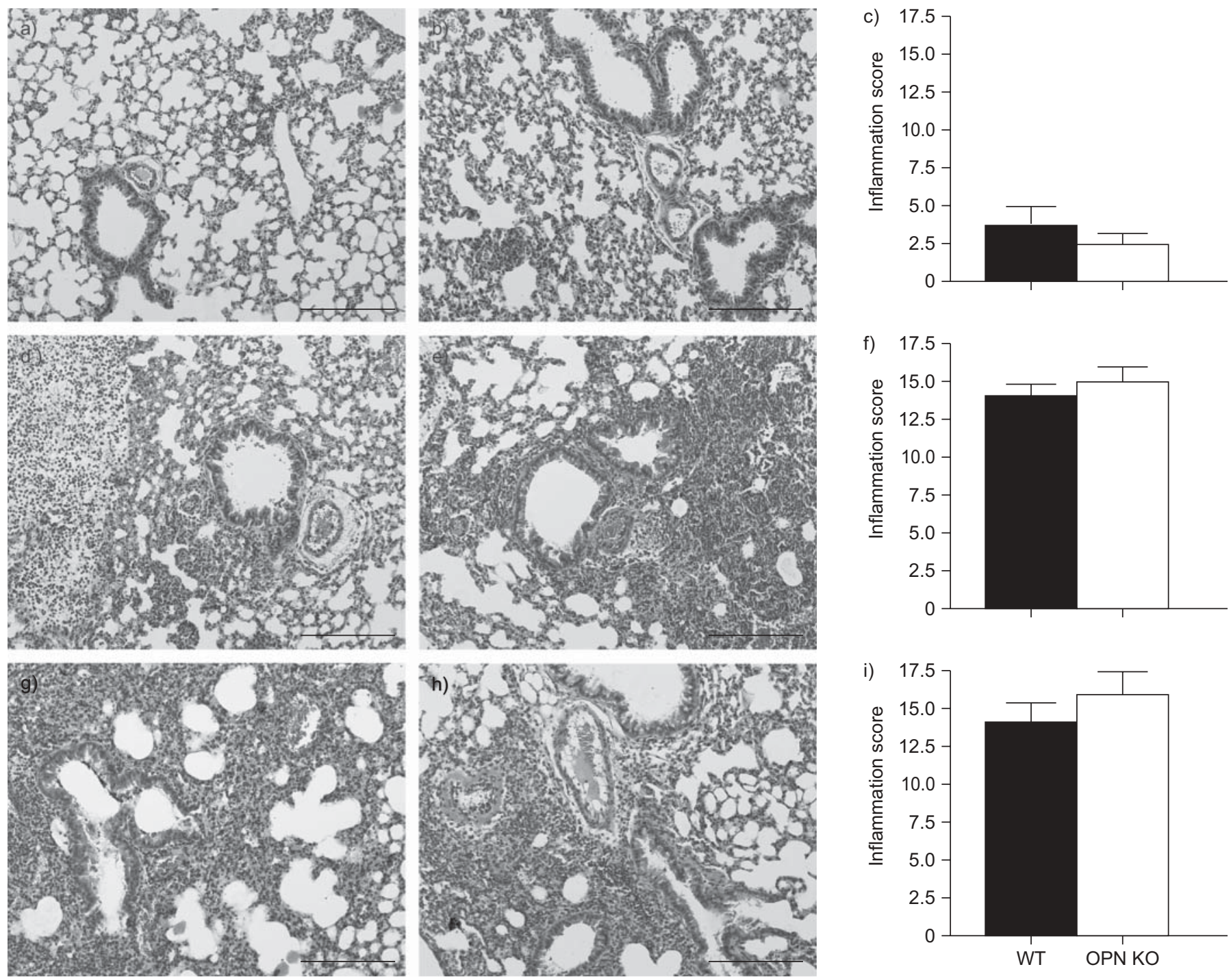

FIGURE 4. Similar lung histopathology. Representative lung histology of wild-type (WT) (a, d, g) and osteopontin (OPN) knockout (KO) (b, e, h) mice at 6 (a-c), 24 (d-f) and $48 \mathrm{~h}(\mathrm{~g}-\mathrm{i})$ after intranasal infection with $10^{4}$ colony-forming units of Klebsiella pneumoniae. The lung sections are representative for eight mice per group per time point. Haemolysin and eosin staining. Scale bars $=1 \mathrm{~mm}$. Pathology scores are presented as mean \pm SEM. $\mathbf{\square}$ : WT; $\square$ : OPN KO; $\mathrm{n}=8$ mice per group.

OPN might additionally be explained by direct effects of OPN on bacterial growth or by altered phagocytosis capacity or phago-lysosomal fusion in OPN KO neutrophils. Therefore, we added OPN to K. pneumoniae and monitored whether bacterial growth was affected. OPN did not alter the growth of this pathogen in vitro (fig. 7a). In addition, phagocytosis capacity and phago-lysosomal fusion were not altered in OPN KO as compared to WT neutrophils (fig. $7 \mathrm{~b}$ and c). Taken together, the observed impairment of bacterial clearance in OPN KO mice cannot be explained by the absence of a bactericidal effect of OPN or by diminished phagocytosis or fusion of phagosomes with lysosomes in OPN KO neutrophils.

\section{DISCUSSION}

The present study is the first to investigate the functional role of OPN during bacterial infection. In accordance with a recent report revealing elevated circulating levels of OPN in patients with bacterial sepsis predominantly suffering from pneumonia [10], we demonstrate elevated local and systemic levels of OPN during Klebsiella pneumonia in mice. OPN was released rapidly into the bronchoalveolar space upon infection of the airways with K. pneumoniae, where it contributed significantly to the early recruitment of neutrophils. Subsequently, OPN KO mice displayed a reduced antibacterial defence at the primary site of infection, as reflected by higher bacterial loads in their lungs, which resulted in a higher mortality rate. The chemotactic function of OPN in the bronchalveolar space towards neutrophils was confirmed in studies using LPS derived from Klebsiella and experiments using rOPN. OPN did not influence antibacterial effector functions of neutrophils. Our data suggest that OPN improves local host defence during Klebsiella pneumonia at least in part by facilitating early neutrophil recruitment. 

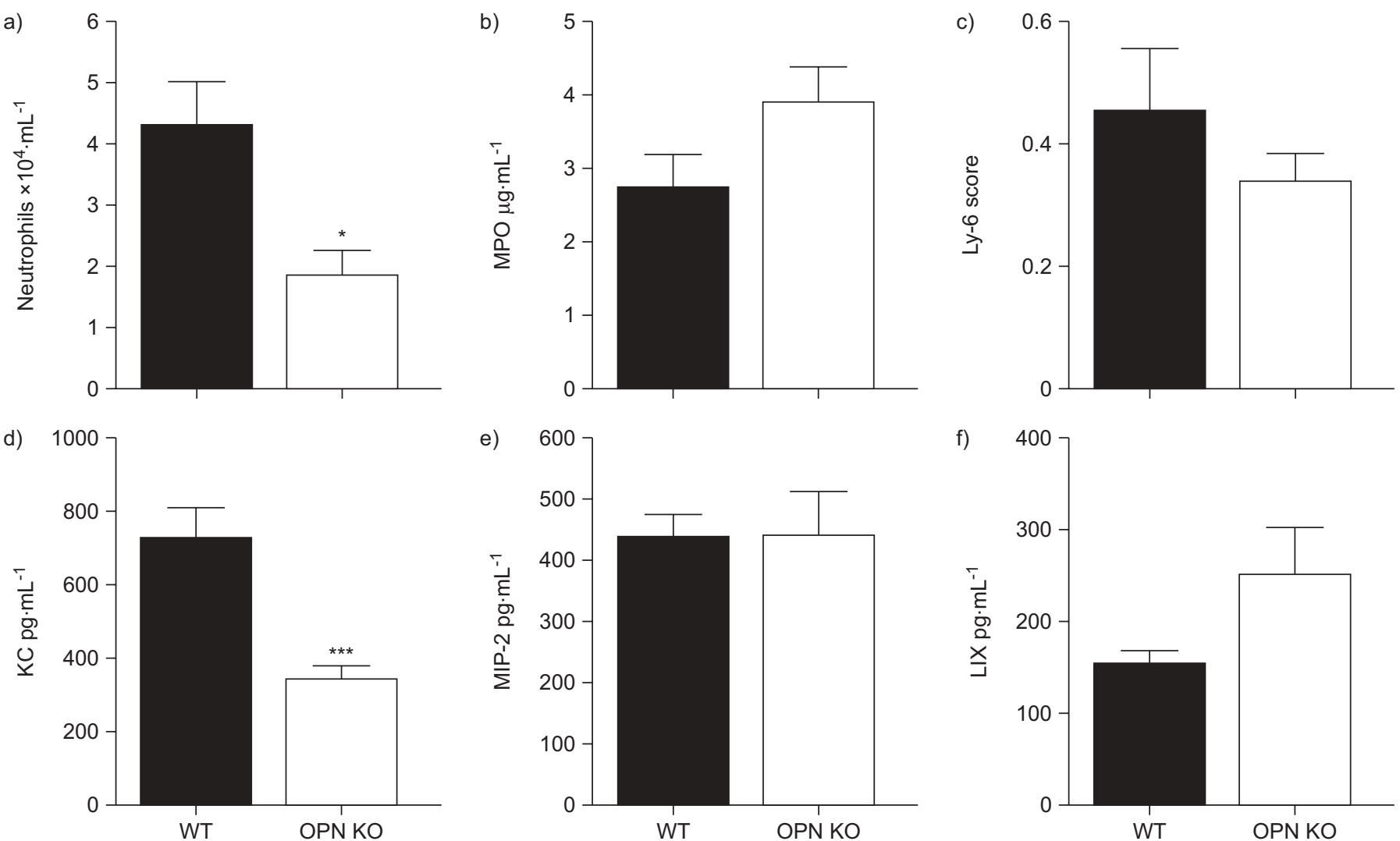

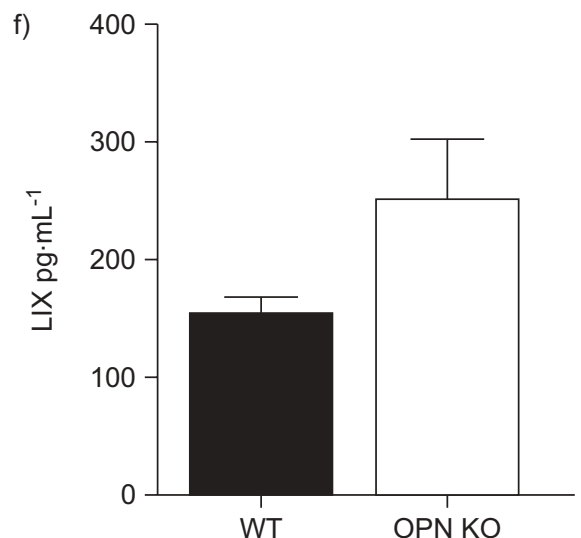

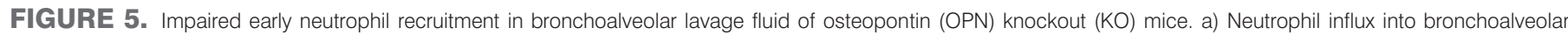

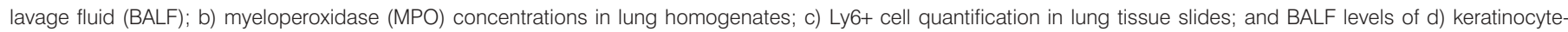

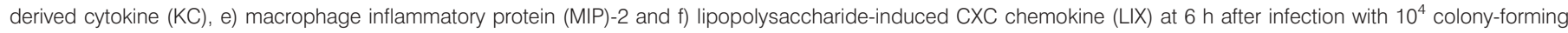
units of Klebsiella pneumoniae. Data are presented as mean \pm SEM; $n=8$ mice per group. *: $p<0.05 ;{ }^{*}$ *: $p<0.001$ compared with wild-type (WT)

Several studies have demonstrated enhanced OPN expression during subacute and chronic pulmonary inflammation $[4,5,7,20]$. However, knowledge of the production of OPN during acute inflammatory diseases is limited. So far, only one study on experimental gram-negative infection, induced by Francisella novicida administered intratracheally, has shown induction of OPN mRNA in lungs of infected mice [21]. We here show that intranasal instillation of viable K. pneumoniae results in a rapid release of OPN into the bronchoalveolar space, whereas OPN levels in lung tissue increased more gradually. Of note, OPN was already detectable in BALF of uninfected mice. Respiratory epithelial cells are a possible source of constitutive OPN levels in BALF: we observed significant spontaneous OPN release upon culturing two distinct murine epithelial cell lines (MLE-12 and MLE-15) without any stimulus added (data not shown). In addition, alveolar macrophages are known to secrete OPN during inflammation [2]. In line, we found profound OPN release by murine alveolar macrophages (MH-S cells) upon stimulation with either Klebsiella LPS or intact K. pneumoniae (data not shown).

In this study, we demonstrated that the increase in OPN levels induced by K. pneumoniae pneumonia served a functional role in host defence against this infection. Although pulmonary bacterial growth was still similar in OPN KO and WT mice at
$6 \mathrm{~h}$ postinfection, enhanced bacterial growth at the primary site of infection was found in the absence of OPN from $24 \mathrm{~h}$ onward. In line, OPN KO mice demonstrated a higher mortality rate. Dissemination of bacteria and organ failure was similar in both groups, suggesting that the difference in pulmonary bacterial loads dominantly determined outcome of the infection. As rapid neutrophil recruitment to the lungs is essential for effective clearance of K. pneumoniae [22], we investigated whether OPN affects this process early during infection. Indeed, we found impaired early neutrophil recruitment into the bronchoalveolar space, but not into lung tissue, in the absence of OPN at $6 \mathrm{~h}$ after infection. As at this early time point, OPN levels were increased in BALF, but not in lung homogenates, compared with uninfected mice, these data suggest that the rapid release of OPN into the bronchoalveolar space upon respiratory tract infection by Klebsiella acts to attract neutrophils into the airways. This suggestion is supported by our experiments in which Klebsiella LPS was used as inciting stimulus (revealing reduced neutrophil influx into BALF of OPN KO mice) and by studies in which the intrapulmonary delivery of rOPN induced neutrophil recruitment into the bronchoalveolar space. Our data confirm and extend earlier data on the role of OPN as a chemoattractant for neutrophils in the liver during alcohol- and Con A-induced hepatitis [23, 24]. Moreover, OPN has been implicated as a chemoattractant for neutrophils in the peritoneal cavity by 

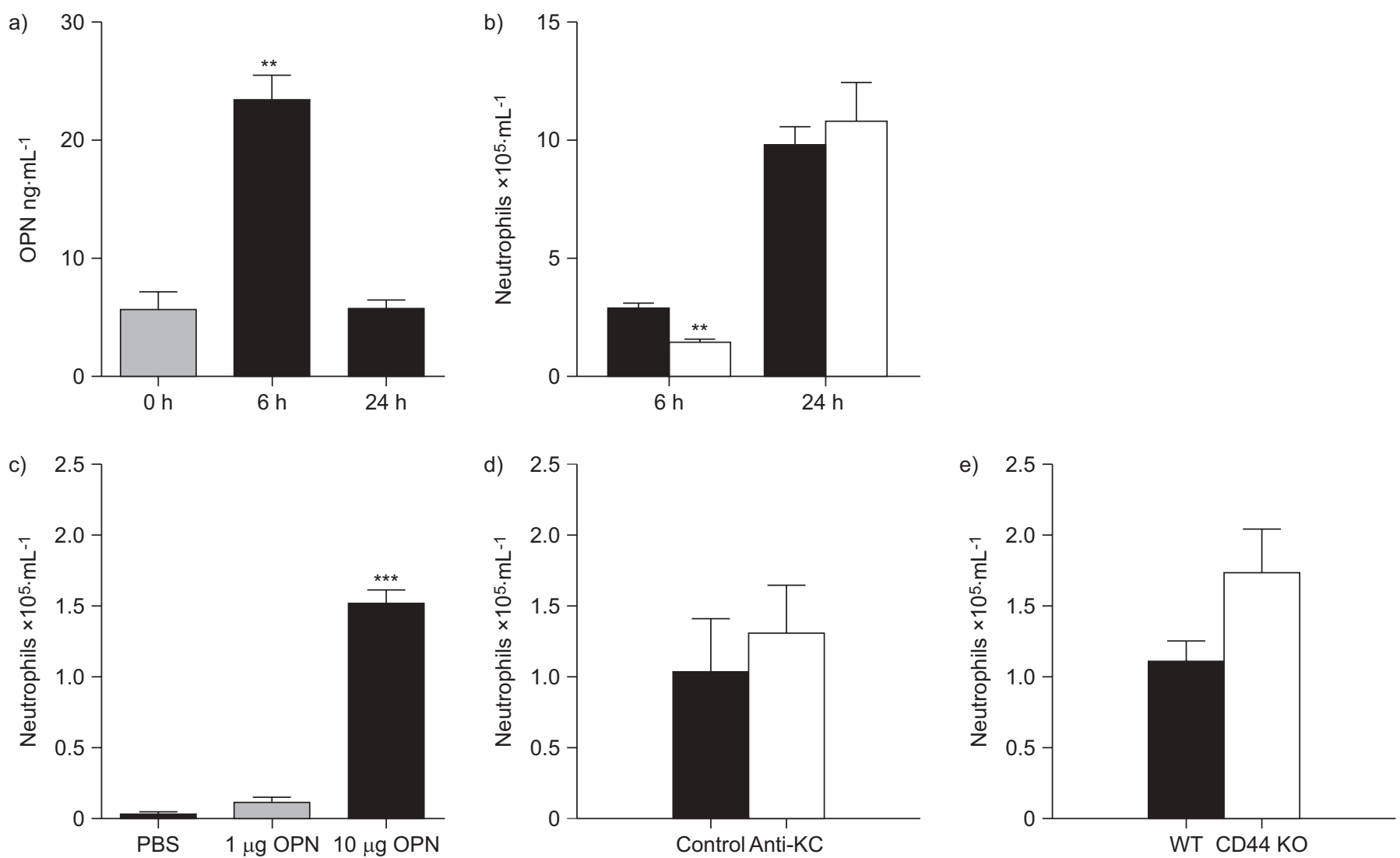

FIGURE 6. Osteopontin (OPN) induces neutrophil recruitment into the bronchoalveolar space. a) OPN levels in bronchoalveolar lavage fluid (BALF) of wild-type (WT; mice and b) neutrophil influx into BALF of WT and OPN knockout (KO; $\square$ ) mice 6 and $24 \mathrm{~h}$ after intranasal administration of $100 \mu \mathrm{g}$ Klebsiella lipopolysaccharide. Data are presented as mean \pm SEM ( $n=6-8$ per group). c) Neutrophil influx into BALF at $6 \mathrm{~h}$ after intranasal PBS, $1 \mu \mathrm{g}$ or $10 \mu \mathrm{g}$ of recombinant OPN (rOPN) administration in WT mice. Neutrophil influx into BALF at $6 \mathrm{~h}$ after intranasal $10 \mu \mathrm{g}$ rOPN administration in d) WT mice that received $50 \mu \mathrm{g}$ keratinolyte-derived cytokine (KC) neutralising antibody or isotype control intraperitoneally $2 \mathrm{~h}$ before rOPN and e) in WT and CD44 KO mice. Data are presented as mean \pm SEM; $\mathrm{n}=5-8$ per group. **: $\mathrm{p}<0.01 ; * * *: p<0.001$.

studies showing an impaired intraperitoneal neutrophil recruitment in OPN KO mice in response to sodium periodate and a robust increase in neutrophil numbers in peritoneal fluid upon local injection of rOPN [25]. In accordance, OPN KO neutrophils were reported to exhibit reduced chemokinesis and chemotaxis towards fMLP, whereas rOPN exerted direct chemotactic effects on neutrophils in vitro [25, 26]. A recent study has indicated that polymerisation of OPN results in an interaction with the $\alpha 9 \beta 1$ integrin on neutrophils, which is essential for the chemotactic function of OPN [27]. Whether
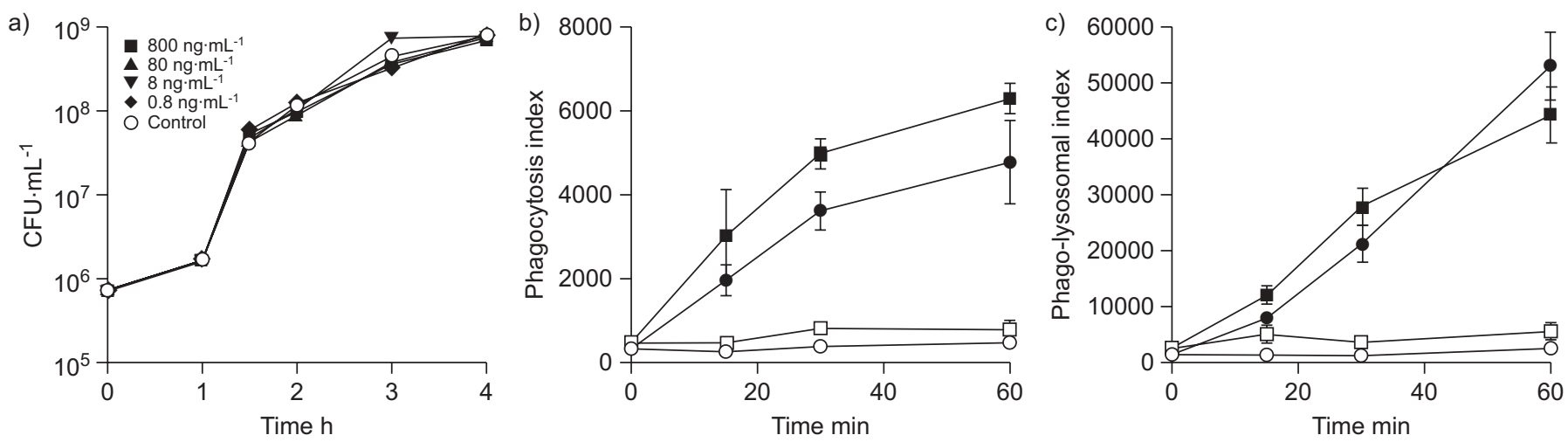

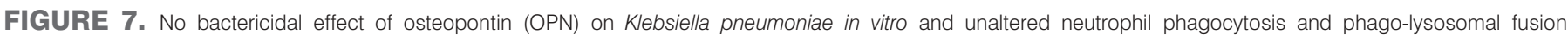

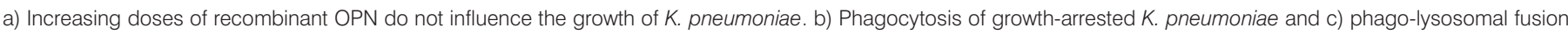

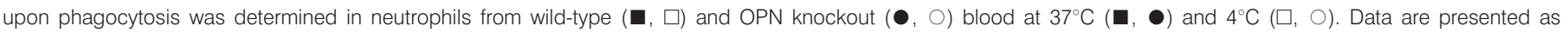
mean \pm SEM; $n=5$ per group. 
this interaction also drives OPN-induced neutrophil migration into the bronchoalveolar space in vivo remains to be established. Our current results argue against an important indirect role for CXC chemokines in the effect of OPN on neutrophil recruitment into the bronchoalveolar space. KC, LIX and MIP-2 have been shown to mediate neutrophil recruitment during gram-negative lung inflammation [28]. Of these, only KC levels were reduced in BALF of OPN KO mice early after infection with Klebsiella, which might have contributed to a diminished neutrophil influx in these animals. However, anti-KC did not impact on influx of these inflammatory cells upon intranasal administration of $\mathrm{rOPN}$, arguing against an intermediate role for $\mathrm{KC}$ in OPN-mediated neutrophil recruitment. Of note, the human and mouse chemokine systems differ and therefore the current results on the role of OPN in a mouse model of pneumonia cannot be extrapolated directly to human respiratory tract infection. In addition, Klebsiella predominantly causes infections in immunocompromised patients; although enhanced OPN expression has been described in patients with immunodeficiency [29]; further research is warranted to establish the functional role of OPN in bacterial infection in the immunocompromised host.

CD44 is an important receptor for $\operatorname{OPN}[2,19]$ and involved in polarisation and chemotaxis of neutrophils [30]. In contrast to macrophages, the expression and localisation of CD44 in neutrophils is independent of OPN expression [25]. We here have demonstrated that rOPN-induced neutrophil recruitment to the bronchoalveolar space was CD44 independent as this response was indistinguishable between CD44 KO and WT mice. Notably, the accumulation of macrophages induced by OPN in vivo was previously found to be dependent on CD44, indicating that OPN exerts chemotactic effects on neutrophils and macrophages by different mechanisms [31]. As Toll-like receptor (TLR) signalling significantly contributes to host defence during K. pneumoniae infection [32-35], a potential interaction between OPN signaling and TLR responses might be of interest. Although the knowledge on such an interaction is very limited, one study has shown that OPN expression by cementoblasts, induced by Porphyromonas gingivalis-derived LPS, which is known to interact with TLR2 and TLR4 [36], was partially inhibited by antibodies against the TLR4/MD2 complex [37].

The enhanced bacterial growth in the absence of OPN could, apart from impaired early neutrophil influx into the bronchoalveolar space, additionally be explained by an altered phagocytosis capacity or phago-lysosomal fusion in OPN KO neutrophils. We found no alterations in the absence of OPN in either of these processes. This result is in accordance with earlier data showing that several antibacterial effector functions of neutrophils are not affected by OPN deficiency [25].

This current model of Klebsiella pneumonia bears resemblance with human pneumonia and sepsis, as it is associated with invasive respiratory tract infection leading to dissemination of infection and distant organ injury, caused by a clinically relevant gram-negative respiratory pathogen. The findings generated in this model of bacterial pneumonia have been considered of relevance for understanding the pathogenesis of pneumonia [38, 39]. Nonetheless, extrapolation to human disease should be made with caution.
In conclusion, we show here for the first time that OPN levels are rapidly elevated in the bronchoalveolar space during $K$. pneumoniae pneumonia, serving a chemotactic function towards neutrophils and thereby an effective innate immune response.

\section{STATEMENT OF INTEREST}

None declared.

\section{ACKNOWLEDGEMENTS}

We thank J. Daalhuisen, M. ten Brink and R. de Beer for expert technical assistance.

\section{REFERENCES}

1 Burwen DR, Banerjee SN, Gaynes RP. Ceftazidime resistance among selected nosocomial gram-negative bacilli in the United States. National Nosocomial Infections Surveillance System. J Infect Dis 1994; 170: 1622-1625.

2 Wang KX, Denhardt DT. Osteopontin: role in immune regulation and stress responses. Cytokine Growth Factor Rev 2008; 19: 333-345.

3 Simoes DC, Xanthou G, Petrochilou K, et al. Osteopontin deficiency protects against airway remodeling and hyperresponsiveness in chronic asthma. Am J Respir Crit Care Med 2009; 179: 894-902.

4 Xanthou G, Alissafi T, Semitekolou M, et al. Osteopontin has a crucial role in allergic airway disease through regulation of dendritic cell subsets. Nat Med 2007; 13: 570-578.

5 Takahashi F, Takahashi K, Shimizu K, et al. Osteopontin is strongly expressed by alveolar macrophages in the lungs of acute respiratory distress syndrome. Lung 2004; 182: 173-185.

6 Berman JS, Serlin D, Li X, et al. Altered bleomycin-induced lung fibrosis in osteopontin-deficient mice. Am J Physiol Lung Cell Mol Physiol 2004; 286: L1311-L1318.

7 O'Regan A. The role of osteopontin in lung disease. Cytokine Growth Factor Rev 2003; 14: 479-488.

8 Kadota J, Mizunoe S, Mito K, et al. High plasma concentrations of osteopontin in patients with interstitial pneumonia. Respir Med 2005; 99: 111-117.

9 Koguchi Y, Kawakami K, Uezu K, et al. High plasma osteopontin level and its relationship with interleukin-12-mediated type $1 \mathrm{~T}$ helper cell response in tuberculosis. Am J Respir Crit Care Med 2003; 167: 1355-1359.

10 Vaschetto R, Nicola S, Olivieri C, et al. Serum levels of osteopontin are increased in SIRS and sepsis. Intensive Care Med 2008; 34: 2176 2184.

11 Schmits R, Filmus J, Gerwin N, et al. CD44 regulates hematopoietic progenitor distribution, granuloma formation, and tumorigenicity. Blood 1997; 90: 2217-2233.

12 Rijneveld AW, Weijer S, Florquin S, et al. Thrombomodulin mutant mice with a strongly reduced capacity to generate activated protein $C$ have an unaltered pulmonary immune response to respiratory pathogens and lipopolysaccharide. Blood 2004; 103: 1702-1709.

13 Tateda K, Moore TA, Newstead MW, et al. Chemokine-dependent neutrophil recruitment in a murine model of Legionella pneumonia: potential role of neutrophils as immunoregulatory cells. Infect Immun 2001; 69: 2017-2024.

14 Knapp S, Wieland CW, van't Veer C, et al. Toll-like receptor 2 plays a role in the early inflammatory response to murine pneumococcal pneumonia but does not contribute to antibacterial defense. J Immunol 2004; 172: 3132-3138.

15 Wiersinga WJ, Wieland CW, Roelofs JJ, et al. MyD88 dependent signaling contributes to protective host defense against Burkholderia pseudomallei. PLoS ONE 2008; 3: e3494. 
16 Miksa M, Komura $\mathrm{H}, \mathrm{Wu}$ R, et al. A novel method to determine the engulfment of apoptotic cells by macrophages using pHrodo succinimidyl ester. J Immunol Methods 2009; 342: 71-77.

17 Tsai WC, Strieter RM, Mehrad B, et al. CXC chemokine receptor CXCR2 is essential for protective innate host response in murine Pseudomonas aeruginosa pneumonia. Infect Immun 2000; 68: 42894296.

18 Ponta H, Sherman L, Herrlich PA. CD44: from adhesion molecules to signalling regulators. Nat Rev Mol Cell Biol 2003; 4: 33-45.

19 Weber GF, Ashkar S, Glimcher MJ, et al. Receptor-ligand interaction between CD44 and osteopontin (Eta-1). Science 1996; 271: 509-512.

20 Pardo A, Gibson K, Cisneros J, et al. Up-regulation and profibrotic role of osteopontin in human idiopathic pulmonary fibrosis. PLoS Med 2005; 2: e251.

21 Roth KM, Oghumu S, Satoskar AA, et al. Respiratory infection with Francisella novicida induces rapid dystrophic cardiac calcinosis (DCC). FEMS Immunol Med Microbiol 2008; 53: 72-78.

22 Ye P, Rodriguez FH, Kanaly S, et al. Requirement of interleukin 17 receptor signaling for lung $\mathrm{CXC}$ chemokine and granulocyte colony-stimulating factor expression, neutrophil recruitment, and host defense. J Exp Med 2001; 194: 519-527.

23 BanERJee A, Apte UM, Smith $\mathrm{R}$, et al. Higher neutrophil infiltration mediated by osteopontin is a likely contributing factor to the increased susceptibility of females to alcoholic liver disease. J Pathol 2006; 208: 473-485.

24 Diao H, Kon S, Iwabuchi K, et al. Osteopontin as a mediator of NKT cell function in T cell-mediated liver diseases. Immunity 2004 21: 539-550.

25 Koh A, da Silva AP, Bansal AK, et al. Role of osteopontin in neutrophil function. Immunology 2007; 122: 466-475.

26 Banerjee A, Lee JH, Ramaiah SK. Interaction of osteopontin with neutrophil $\alpha 4 \beta 1$ and $\alpha 9 \beta 1$ integrins in a rodent model of alcoholic liver disease. Toxicol Appl Pharmacol 2008; 233: 238-246.

27 Nishimichi N, Higashikawa F, Kinoh HH, et al. Polymeric osteopontin employs integrin $\alpha 9 \beta 1$ as a receptor and attracts neutrophils by presenting a de novo binding site. J Biol Chem 2009, 284: 14769-14776.

28 Craig A, Mai J, Cai S, et al. Neutrophil recruitment to the lungs during bacterial pneumonia. Infect Immun 2009; 77: 568-575.

29 Chagan-Yasutan H, Saitoh H, Ashino Y, et al. Persistent elevation of plasma osteopontin levels in HIV patients despite highly active antiretroviral therapy. Tohoku J Exp Med 2009; 218: 285-292.

30 Alstergren $\mathrm{P}$, Zhu B, Glogauer M, et al. Polarization and directed migration of murine neutrophils is dependent on cell surface expression of CD44. Cell Immunol 2004; 231: 146-157.

31 Marcondes MC, Poling M, Watry DD, et al. In vivo osteopontininduced macrophage accumulation is dependent on CD44 expression. Cell Immunol 2008; 254: 56-62.

32 Branger J, Knapp S, Weijer S, et al. Role of Toll-like receptor 4 in gram-positive and gram-negative pneumonia in mice. Infect Immun 2004; 72: 788-794.

33 Bhan U, Lukacs NW, Osterholzer JJ, et al. TLR9 is required for protective innate immunity in Gram-negative bacterial pneumonia: role of dendritic cells. J Immunol 2007; 179: 3937-3946.

34 Cai S, Batra S, Shen L, et al. Both TRIF- and MyD88-dependent signaling contribute to host defense against pulmonary Klebsiella infection. J Immunol 2009; 183: 6629-6638.

35 Schurr JR, Young E, Byrne P, et al. Central role of toll-like receptor 4 signaling and host defense in experimental pneumonia caused by Gram-negative bacteria. Infect Immun 2005; 73: 532-545.

36 Bainbridge BW, Coats SR, Darveau RP. Porphyromonas gingivalis lipopolysaccharide displays functionally diverse interactions with the innate host defense system. Ann Periodontol 2002; 7: 29-37.

37 Nociti FH Jr, Foster BL, Barros SP, et al. Cementoblast gene expression is regulated by Porphyromonas gingivalis lipopolysaccharide partially via toll-like receptor-4/MD-2. J Dent Res 2004; 83: 602-607.

38 Strieter RM, Belperio JA, Keane MP. Cytokines in innate host defense in the lung. J Clin Invest 2002; 109: 699-705.

39 Mizgerd JP. Acute lower respiratory tract infection. N Engl J Med 2008; 358: 716-727. 